Meta

Journal des traducteurs

Translators' Journal

\title{
Problèmes terminologiques de la version grecque des textes legislatifs des communautés européennes
}

\section{Vassilis Koutsivitis}

Volume 36, numéro 1, mars 1991

La terminologie dans le monde : orientations et recherches

URI : https://id.erudit.org/iderudit/003347ar

Aller au sommaire du numéro

Éditeur(s)

Les Presses de l'Université de Montréal

ISSN

0026-0452 (imprimé)

Découvrir la revue

Citer cet article

Koutsivitis, V. (1991). Problèmes terminologiques de la version grecque des textes legislatifs des communautés européennes. Meta, 36(1), 284-290. d'utilisation que vous pouvez consulter en ligne. 


\title{
PROBLÈMES TERMINOLOGIQUES DE LA VERSION GRECQUE DES TEXTES LÉGISLATIFS DES COMMUNAUTESS EUROPÉENNES
}

\author{
VASSILIS KoUTSIVITIS \\ Commission des Communautés européennes*, Bruxelles, Belgique
}

Le texte législatif communautaire est composé de trois couches: le texte libre, les formules standardisées et les termes techniques!.

Par texte libre, on entend discours courant, l'énonciation spontanée qui n'obéit pas aux règles régissant la partie normalisée du texte.

La normalisation du discours législatif communautaire, qui découle du caractère contraignant de ces textes, conduit en effet à la création des correspondances préétablies et répétitives, qu'on peut désigner par l'appellation générique formules standardisées. Il s'agit d'énoncés qui ont toujours un sens mais qui se trouvent à l'état figé, et c'est pour cette raison qu'ils doivent être transposés de manière consacrée. Leur rigidité est due soit à des normes rédactionnelles, soit au caractère protocolaire, solennel et contraignant des actes législatifs.

La troisième couche est constituée des termes techniques. Il s'agit de mots ou syntagmes qui correspondent à des notions bien définies, structurées et hiérarchisées. Ils relèvent généralement du transcodage mais ils n'excluent pas toujours la créativité ni la contextualité, en fonction de leur origine et de leur finalité. La partie la plus frappante de la terminologie communautaire est une nouvelle nomenclature composée de lexèmes qu'on ne rencontre pas très souvent en dehors des textes en question, ou qui sont utilisés avec une acception bien spécifique.

Un praticien et théoricien très attentif de la terminologie communautaire a subdivisé celle-ci en quatre catégories selon les textes d'origine et la nature des termes en question ${ }^{2}$ :

1) la terminologie «intouchable» tirée des Traités et du droit primaire,

2) la terminologie mi-libre liée au fonctionnement des institutions,

3) la terminologie portant sur l'objet technique des traités,

4) la terminologie en rapport avec l'intégration européenne.

Quant à nous, nous procéderons dans cet article à l'analyse des termes techniques communautaires en nous référant surtout à leur fonction dans les textes et à leur place dans le processus traductif, et en mettant l'accent sur les problèmes de la version grecque.

\section{TERMINOLOGIE COMMUNAUTAIRE ET LANGAGE COURANT}

Les termes techniques de la législation communautaire doivent, comme tous les termes juridiques, rccouvrir des notions précises. Le même terme doit être utilisé pour la même notion sans interprétation extensive ou restrictive. Les termes communautaires doivent en plus ne pas prêter à confusion avec des termes de droit interne des États membres de la Communauté. 
Mais cette uniformité terminologique au niveau communautaire n'est pas acquise sans pertes au niveau des langues nationales. C'est de l'ordre juridique communautaire que surgit la nécessité d'un langage unique, d'un jargon communautaire.

...Pour éviter le risque de confusion entre l'acception nationale et l'acception communautaire d'un même mot, il est admis de créer une terminologie communautaire ( $p$. ex. «praticien de l'art dentaire» pour «dentiste»); toutefois, cette latitude est utilisée avec discrétion et en outre elle est tempérée par le respect des particularités linguistiques ou juridiques : il peut être renoncé à l'équivalence mot-à-mot dans l'intérêt de la clarté̉.

Ainsi, l'article 189 du traité CEE énumère et définit les actes que peuvent approuver les institutions communautaires: règlement - directive — décision recommandation - avis. Il s'agit de termes qui sont le plus souvent chargés d'un tout autre contenu dans les ordres juridiques des États membres et qui en plus peuvent signifier autre chose au titre du traité instituant la Communauté européenne du charbon et de l'acier.

L'existence d'un mot-terme au sein du jargon communautaire n'implique pas automatiquement que ce lexème perd son droit d'existence en tant que mot commun.

Ainsi, dans les articles 145 et 155 , le mot décision ne désigne pas le terme technique de l'article 189, mais couvre le même champ sémantique que la langue courante.

La langue du droit, comme toute langue de spécialité où cohabitent terminologie et texte libre, ne peut pas atteindre la monoréférentialité des langues artificielles ou des langages des ordinateurs. Il y a toujours des effets de subjectivité qui échappent à la volonté de l'auteur et qui sont la source de toutes sortes de déviations sémantiques.

Les termes sont aussi caractérisés et distingués des lexèmes communs par une différenciation très poussée, qui obéit à des règles strictes. Le traducteur spécialisé doit rester vigilant et essayer de déceler si l'auteur de l'original emploie un mot par goût stylistique ou par contrainte terminologique. Dépister cette volonté et parvenir ou échouer à s'y conformer conditionnent dans une large mesure la réussite de la traduction.

Voici un exemple à éviter: le mot metro, - $a(=$ mesure, $-\mathrm{s})$ est employé dans la version grecque du traité CEE comme correspondant à six termes français : mesure (art. 5), réglementation (art. 7), actions (art. 8, par. 2), réalisations (art. 8, par. 7), disposition (art. 11) et moyens (art. 89). Nous ne prétendons pas que la synonymie et la polysémie sont chassées une fois pour toutes du royaume de la terminologie. Mais il serait difficile de nier que, dans ce cas, le texte grec n'atteint pas un niveau d'équivalence sémantique suffisant pour un instrument juridique.

Par contre, nous trouvons plusieurs exemples où le traducteur n'est pas tombé dans le piège de la banalisation des termes et a respecté la technicité du langage. Citons-en deux. Le premier concerne les mots origine et provenance d'un produit. La phrase $\ll[\ldots]$ produits qui sont originaires des États membres [...]» (art. 9, par. 2) est traduite littéralement «[...] proïonta katagogis kraton melon [...]». Le terme katagogi (= origine) est employé en langue courante uniquement pour des personnes. On serait donc tenté de dénoncer ce barbarisme et de proposer le terme habituel proelefsi (= provenance), mais celui-ci arrive tout de suite après, «ainsi qu'aux produits en provenance de pays tiers [...]» avec une signification technique clairement distincte.

En effet, la notion d'origine est plus étroite que celle de provenance et elle est définie dans le cadre du règlement (CEE) 802/68 du Conseil du 27 juin 1968, JOCE no $\mathrm{L}$ $148 / 68$, qui stipule entre autres: art. 4 :

Sont originaires d' un pays les marchandises entièrement obtenues dans ce pays. 
art. 5:

Une marchandise dans la production de laquelle sont intervenus deux ou plusieurs pays est originaire du pays où a eu lieu la dernière transformation ou ouvraison substantielle, économiquement justifiée, effectuée dans une entreprise équipée à cet effet et ayant abouti à la fabrication d' un produit nouveau ou représentant un stade de fabrication important.

Pour étayer toutefois les quelques doutes que nous avons exprimés sur la terminologisation parfaite du matériel lexical des langues de spécialité, nous signalons en passant l'emploi parallèle et sans distinction apparente des expressions importations originaires et importations en provenance dans l'article 133, par. 1 et 5.

\section{DÉFINITIONS ET HIÉRARCHISATION}

Le législateur communautaire s'efforce en principe d'employer des termes clairs et précis dans leur acception usuelle. Néanmoins, les craintes ou les risques de confusion l'amènent souvent à définir les termes qu'il emploie; et parfois, il ne se contente pas d'une définition théorique, mais il la complète par une énumération restrictive des objets auxquels il se réfère.

1. Le marché commun s'étend à l'agriculture et au commerce des produits du sol, de l'élevage et de la pêcherie, ainsi que les produits de première transformation qui sont en rapport direct avec ces produits [...]. Les produits qui sont soumis aux dispositions des articles 39 à 46 inclus sont énumérés à la liste qui fait l'objet de l'annexe II du présent traité [...] (art. 38)

Ainsi, quand le traité se réfère aux produits agricoles, il s'agit des produits qu'il définit comme tels, indépendamment de n'importe quelle autre classification.

Quelquefois, cette délimitation du contenu notionnel d'un terme n'est pas donnée d'une manière explicite mais indirectement, soit à travers le contexte, soit à l'aide de notes. Ces précisions aident beaucoup le traducteur et lui évitent des pièges.

À propos, par exemple, de la délimitation des termes évaluation et appréciation qui, en langue courante, seraient considérés comme synonymes, un rédacteur de textes communautaires explique:

After much discussion it became evident that "evaluation" involved the concept of measurement, which was not the case for "assessment". It was finally agreed to use in French the word "appreciation" 4

Les termes d'une langue technique développent entre eux des relations très précises parmi lesquelles le lien hiérarchique est fondamental. Ainsi, de toutes les instances communautaires créées par le traité CEE, quatre seulement, les principales, sont désignées par le terme institution: le Parlement, le Conseil, la Commission et la Cour de Justice (art. 4, par. 1). Pour les autres, le terme organe est d'usage.

En grec, cette relation a été troublée par le fait qu'on a traduit institution par organo (= organe). Par conséquent, on a dû rétablir cette distinction qualitative par une périphrase: kyria $i$ thesmika organa (= organes principaux ou institutionnels) pour institutions et defterevonta $i$ epikourika organa (= organes secondaires ou auxiliaires) pour organes.

\section{UNE NOUVELLE NOMENCLATURE}

Pour répondre aux objectifs qu'il se fixe, le jargon communautaire crée également une nouvelle nomenclature. Ainsi, l'adjectif national, qui signifie ici d' un État membre et qui s'oppose souvent à l'adjectif communautaire, peut accompagner presque tous les substantifs en créant des syntagmes qui, dans la langue courante, n'auraient pas de sens 
ou éveilleraient des connotations non voulues (p. ex. gouvernement national, politique nationale, etc.)

Les traducteurs grecs ont essayé, et ils essaient toujours, mais dans une moindre mesure, d'éviter ces pièges en employant la périphrase équivalente d'un État membre. Voir p. ex. l'art. 48, par. $3 \mathrm{c}$. du traité CEE travailleurs nationaux = ergazomenoi ypikooi aftou tou kratous melous (5 mots pour 1!). Force est de constater que le terme correspondant à national (= ethnikos) s'impose de plus en plus dans tous les cas.

Cette création de notions nouvelles est continuelle et pléthorique :

Mais en plus, les institutions internationales sécrètent des notions administratives qui ont parfois leur équivalent exact dans un seul des pays membres et dans une seule langue et qui n'ont qu'une équivalence approximative avec les notions analogues, mais non identiques, des autres pays membres et des autres langues. Ou encore, elles sécrètent des notions qu'elles veulent nouvelles, sans équivalence exacte dans aucun des pays ni aucune des langues. Je vous donne tout de suite un exemple. Chaque institution des Communautés européennes est dotée d'un contrôleur financier, dont le rôle s'apparente à celui du comptable des dépenses engagées dans les administrations belges. Mais dans aucun des dix pays membres il ne se trouve de fonctionnaire dont le rôle soit identiquement celui du contrôleur financier des institutions européennes. En revanche, les Communautés ne disposent pas d'inspecteurs des finances comme en France et en Belgique. Sous la forme où on le trouve dans les institutions européennes, le contrôle financier est une notion nouvelle et ce serait une faute de traduction que de l'appeler comptabilité des dépenses engagées. Pour traduire la dénomination du contrôleur financier, il ne pourra être question de chercher dans le droit allemand, britannique, néerlandais, italien ou danois des notions qui n'y existent pas et des termes qui recouvriraient des notions inexistantes au plan national ${ }^{5}$.

Un autre problème auquel est malheureusement assez souvent confronté le traducteur d'une famille de textes consiste à ce qu'un terme s'impose dans la langue cible a posteriori, à retardement, c'est-à-dire non dès sa première apparition, mais lors d'une apparition ultérieure. Dans ce cas, et quand en plus il s'agit de textes normatifs, à part les remords du traducteur, nous avons le problème du respect ou du non-respect des antécédents et d'éventuels rectificatifs.

Ainsi formation professionnelle (art. 41, a) a été traduit dans la version grecque du traité CEE par epangelmatiki ekpaidefsi. On peut supposer que le traducteur a préféré le terme ekpaidefsi (= éducation, enseignement) au terme katartisi (= formation), qui s'est imposé par la suite dans les textes communautaires, pour deux raisons principales:

a) le terme ekpaidefsi est beaucoup plus clair et élégant en grec que le correspondant exact de formation, et

b) le terme katartisi ne correspondait à l'époque en Grèce à aucune situation structurée, mais plutôt à une pratique encore mal connue et peu organisée.

Néanmoins, l'expérience a démontré que la réalité communautaire exigeait l'existence de deux termes bien distincts, et, en effet, la langue (et la réalité) grecque a dû s'y adapter6.

\section{TERMINOLOGIE ET LANGUE CIBLE}

Un cas qui mérite l'attention dans ce cadre est celui des emprunts. Ces corps étrangers - du moins au moment de leur apparition - dans la langue d'arrivée sont encore plus flagrants en grec que dans les langues romanes et germaniques, à cause des difficultés graphiques - qu'on essaie de surmonter par une translittération toujours anarchique - phonologiques et morphologiques.

Dans le traité CEE, nous avons l'exemple du terme dumping (article 91) 7 . Ce mot anglais est repris tel quel dans toutes les langues communautaires et il est trop tard (entré 
en français en 1904) pour se poser des questions sur sa traduisibilité (!). De toute façon, pour le grec, il y a la proposition du terme ypotimitismos ${ }^{8}$, mais vu l'absence en Grèce de structures analogues aux différentes commissions de terminologie existant en France, on ne risque pas de voir ce mot remplacer dumping, même dans le cas (improbable) où les autres conditions requises seraient réunies.

La transposition de termes du langage communautaire exerce apparemment une influence importante sur la structure de la langue d'arrivée, surtout au niveau sémantique, mais également sur le plan syntaxique et morphologique.

Ainsi, le caractère multinational de la construction communautaire engendre une pléthore de pluriels pour des notions qui auparavant étaient conçues - du moins en grec - uniquement au singulier: c'est le cas par exemple de la politique économique. C'est pour cela que, quand le traducteur se confronte à la phrase coordonner les politiques économiques des États membres, sa première réaction est d'ignorer le pluriel et de traduire syntonismo tis oikonomikis politikis ton kraton melon (art. 3, alinéa g du traité CEE) ou d'essayer de l'esquiver par syntonizoun kathe oikonomiki tous politiki (art. 6 , par. 1) (= toute politique économique). Mais la force de la répétition combinée avec le besoin de précision de plus en plus ressenti ont eu comme résultat l'apparition et la consécration d'une série de pluriels de substantifs ignorés par le grec autochtone: politikes, draseis, stratigikes, ypodomes, praktikes, etc. (politiques, actions, stratégies, infrastructures, pratiques...).

Dans d'autres cas, le texte exige de la part du traducteur grec une attention plus que soutenue, voire sa circonspection, pour qu'il évite les pièges tendus par les faux amis.

«logistique» n'est pas logistiki (= comptabilité)

«apocalyptique» n'est pas apokalyptikos (= révélateur)

ni «symptomatique», symptomatikos (= accidentel)

ni «énergétique», energitikos (= énergique), et ainsi de suite.

\section{ASPECTS MÉTALINGUISTIQUES}

Comme si les contraintes linguistiques ne lui suffisaient guère, le traducteur qui essaie de transposer un terme technique est souvent en proie à des nuances dont le caractère sort du système de la langue. Il s'agit surtout de considérations politico- ou sociolinguistiques.

Les qualificatifs utilisés, p. ex. pour nommer les régions pauvres de la Communauté économique européenne, varient: a) régions sous-développées (art. 80), b), régions moins développées (art. 130a), c), régions moins favorisées (préambule) et d) défavorisées (art. 42). Ce dernier adjectif n'est pas utilisé dans le traité CEE pour les régions, mais pour des exploitations agricoles; cependant, son emploi est courant dans les textes de la politique régionale commune (cf. Règlement 1932/84, JOCE no L180/84). Leur traduction littérale en grec est: a) ypanaptyktes, b) ligotero anaptygmenes, c) ligotero evnoïmenes et d) meionektikes (-ouses) perifereies. La variation de ces termes n'est pas fonction d'une nécessité terminologique et ne correspond pas à une typologie (comme, régions centrales et régions périphériques, ou régions avec retard structurel et régions de déclin industriel) mais est due à des facteurs métalinguistiques et constitue une tentative d'éviter des expressions chargées politiquement ou socialement. La preuve est que le terme le plus «dur» parmi les quatre (sous-développées) a depuis longtemps disparu de la terminologie communautaire.

Comment le traducteur peut-il réagir face à ces différentes appellations de la même situation? Première solution, il essaie de rendre à chacune son équivalent linguistique exact, dans la mesure où la langue cible le permet, en respectant ainsi la volonté 
stylistique de l'auteur. Deuxième solution, il les traduit toutes de la même façon en employant le terme le plus pertinent en langue d'arrivée, en rétablissant l'unicité et la cohésion terminologiques qui manquent à l'original. À notre avis, les deux façons sont en principe correctes.

La tâche du traducteur devient très délicate quand il s'agit de termes politiquement sensibles. Nous trouvons un tel exemple important, car son enjeu est fondamental, en lisant l'article 222 du traité CEE qui concerne le régime de la propriété; le texte français se lit ainsi :

Le présent traité ne préjuge en rien le régime de la propriété dans les États membres.

Cette phrase peut être interprétée de deux façons: une dynamique qui se réfère à l'avenir, à l'évolution et une statique qui concerne la situation présente. Sans vouloir entrer dans une analyse purement juridique, le texte dans son ensemble nous amène à penser que le législateur faisait allusion aux deux possibilités, que l'expression française peut englober. Mais on constate que si la langue d'arrivée n'offre pas un pareil luxe, le traducteur doit choisir l'une ou l'autre direction:

GR : [...] den prodikazei me kanena tropo [...]

$\mathrm{DE}:[\ldots]$ lässt unberührt $[\ldots]$

$\mathrm{EN}:[\ldots]$ shall in no way prejudice $[\ldots]$

$\mathrm{NL}:[\ldots]$ laat onverlet $[\ldots]$

DA : [...] berores ikke $[\ldots]$

IT : [...] lascia del tutto impregiudicato $[\ldots]$

ES : [...] no prejuzga en mode alguno [...]

PT $:[. .$.$] en nada prejudica [. .$.

La traduction grecque est celle qui insiste plus que toutes les autres sur la possibilité d'évolution du régime de propriété dans les États membres.

La terminologie est une couche aussi bien particulière qu'essentielle des textes législatifs communautaires. Elle a ses propres caractéristiques, notamment la rigueur notionnelle et la structure hiérarchisée et, par conséquent, ses propres exigences pour le traducteur.

Elle n'évolue pas in vitro, mais elle est en constante interaction avec le texte libre et les formules standardisées, et elle est également affectée par la réalité extralinguistique, situation qui crée des difficultés, mais aussi des possibilités traductives supplémentaires.

Son influence sur la langue cible est importante.

Les termes techniques des actes communautaires sont en somme un champ à part entière d'actualisation du processus traductif et ils doivent être envisagés en tant que tel par le traducteur.

\section{NOTES}

* L'auteur exprime dans cet article ses idées personnelles qui n'engagent en rien la CCE.

1. Il s'agit d'une des conclusions principales de notre thèse (KOUTSIVITIS, 1988). Le présent article est d'ailleurs en grande partie une synthèse du chapitre de ladite thèse sur les termes techniques (pp. 307-343).

2. Voir R. GOFFIN (1986: 7-11)

3. BAUER-BERNET, H. (1982: 191-192)

4. Voir W. HUNTER $(1985: 38)$ 
5. EVENS, V. (1987: 45)

6. Comme s'il fallait des preuves que la réalité traductologique et terminologique est riche en rebondissements et que le droit comme la linguistique ne sont pas des sciences exactes, les instances communautaires ont commencé récemment à admettre que la formation professionnelle pouvait dans un sens large englober l'éducation: «[...] une lecture très littérale des termes du traité de Rome a été longtemps un argument «théologique» pour exclure l'éducation du champ de compétences de la Communauté (alors que la formation professionnelle s'y trouve)». MARIN, M. (1988): «Au-delà des querelles théologiques», Campus, supplément au Monde, no 13403, 3 mars, p. 5.

7. Selon le Petit Robert, éd. 1987, «Pratique qui consiste à vendre sur les marchés extérieurs à des prix inférieurs à ceux qui sont pratiqués sur le marché national (et parfois aux prix de revient).»

8. VLACHOS, A. (1983). Voir aussi antiypotimisi ou anthypotimisi pour antidumping dans: DELTIO, (1986:110)

\section{BIBLIOGRAPHIE}

BAUER-BERNET, H. (1982): «Le multilinguisme du droit de la Communauté européenne», GÉMAR, J.-C. (dir.), Langage du droit et traduction, Québec, Linguatech, Conseil de la langue française, pp. 187-205.

DELTIO (1986): Deltio epistimonikis orologias kai neologismon, Grafeio epistimonikon oron kai neologismon, Akadimia Athinon.

EVENS, V. (1987) : «La traduction administrative en milieu national et international», Babel, 1, pp. 39-47.

GOFFIN, R. (1986): «Les ouvrages terminographiques des Communautés européennes», Terminologie et traduction, 3, pp. 3-13.

HUNTER, W. (1985): «The long Voyage of Man's Discovery of Language», Terminologie et traduction, 2, pp. $37-42$.

KOLAITIS, M. (1976): Greek-English Dictionary of Pure and Applied Mathematics, T.E.E., Athènes, (v. notamment l'introduction, en grec, pp. I-CI).

KOUTSIVITIS, V. (1988): «La traduction juridique. Étude d'un cas: la traduction des textes législatifs des Communautés européennes et en particulier à partir du français vers le grec», Thèse de doctorat, Université de la Sorbonne nouvelle - Paris III (non publiée).

SHUMACHER, N. (1988): «Les métaphores du discours européen», Langage et l'Homme, 3, fasc. 1, n॰66, pp. 40-47.

VLACHOS (1983): VLACHOS, A., O exellinismos ton xenon lexeon, I KATHIMERINI, 17-24 avril 1983. 